\title{
Hierarchy of States and the Dirac Equation in the Quantum Hall Effect
}

\author{
Keshav N. Shrivastava, Fellow, IACSIT
}

\begin{abstract}
The hierarchy of states is developed with increasing the value of $l$, the orbital angular momentum, is now called "Shrivastava's hierarchy of fractional states". We have examined the odd denominator states as extended to a hierarchy of continued fraction which describes a very large abundance of fractional states. The heirarchy developed by random numbers $\mathrm{m}$ and $p_{i}(i=1,2, \ldots, 4)$ by using the continued fraction is known as the Haldane's hierarchy. We find that the predictions of the Dirac equation agree with the idea of fractional charges. We have introduced the combination of spin and orbital quantum numbers, including the negative sign for spin, in such a way that there occur fractional charges through the Bohr magneton. This leads to doubling of eigen values so that we define an additional matrix the properties of which are important when magnetic field is present. There is a spin-charge coupling so that spin $1 / 2$ particle can have the zero or one charge. The Dirac equation can accommodate not only charges of 0 and $\pm e$ but also fractional values such as $1 / 3$ and 2/3.
\end{abstract}

Index Terms - Dirac equation, fractional charges, hierarchy, angular momentum.

\section{INTRODUCTION}

Recently, in three papers, we have laid down the basically correct theory of the quantum Hall effect [1]-[3]. It has been found [4], [5] in the Hall effect that the transverse conductivity as a function of magnetic field shows plateaus at certain fractions of $e^{2} / h$. The measurement of the resistivity in the plateau region can be performed with very high precision. In the absence of scattering processes, at low temperatures and high fields, the classical Hall current is described by the fraction,

$$
I_{x}=\frac{n_{s} e V_{y}}{B}
$$

where $V_{y}$ is the applied voltage, e the charge of the electron, B the magnetic field and $n_{s}$ the surface carrier density,

$$
n_{s}=v N
$$

where $v$ is a filling factor and

$$
N=e B / h c
$$

is the degeneracy factor per unit area obtained from the shift

Manuscript received March 30, 2013; revised May 30, 2013

Keshav N. Shrivastava is with the University of Malaya, Kuala Lumpur, and with the University of Hyderabad, Hyderabad 500046, India (e-mail: keshav1001@yahoo.com). of the oscillator wave function upon the application of magnetic field. Substituting (2) and (3) in (1) we find that,

$$
I_{x}=v \frac{e^{2} V_{y}}{h c}
$$

which means that the conductivity is given by,

$$
\sigma_{x y}=v \frac{e^{2}}{h c}
$$

From (2) and (3) we find that,

$$
v=\frac{n_{s}}{e B / h c}
$$

which means that the carrier density and the field are adjusted in such a way that the filling factor $v$ of the energy levels is an integer. From the occurrence of plateau in the Hall conductivity for $v=1$, von Klitzing et al. [4] were able to get the value of $h / e^{2}$. The plateaus in the Hall resistivity as a function of magnetic field were observed at many different fractions in addition to integer values.

\section{HALDANE'S HIERARCHY}

Haldane [6] suggested that the fractional filling factors occur according to a hierarchy which is built from numbers $\mathrm{m}$ and $p_{i}(i=1,2$ or 3$)$ determined from a continued fraction in which the number of terms determine the number of fractions. These numbers $\mathrm{m}$ and $p_{i}$ are not derived from any physical properties but are integers. Since $\mathrm{m}$ and $p_{i}$ are not related to the physics of electrons, atoms or crystals, these are not going to lead to a physics related filling factor. In any case, Haldane suggested,

$$
v=\frac{1}{m}
$$

where $m$ is an odd integer. The daughter states are produced by taking,

$$
v=\frac{1}{m \pm \frac{1}{p_{1}}}
$$

where $p_{1}$ is an integer. The grand daughter states are given by $v=\frac{1}{m \pm \frac{1}{p_{1} \mp \frac{1}{p_{2}}}}$ and the grate-grand daughter states are 


$$
v=\frac{1}{m \pm \frac{1}{p_{1} \pm \frac{1}{p_{2} \pm \frac{1}{p_{3}}}}}
$$

which can be continued up to $p_{\mathrm{n}}$. The present family members are determined by $n$. For $m=1$, the first member is $v=1$, for $n=1, m=1$ and $p_{1}=2$ has two daughters $v=2 / 3+$ and 2 depending on the sign in (8). In the extreme quantum limit, $v<1$, so that we may leave out the $v=2$ - which occurs at lower fields. For $n=2, m=1, p_{1}=p_{2}=2$, the continued fraction gives $5 / 7++, 5 / 3+-, 3 / 5-+$ and 3-- and for $n=3, p_{1}=p_{2}=p_{3}=2$ the fractions are 12/17+++, 8/13+-+, 8/11-++, 4/7--+, 12/7++-, $8 / 3+--, 8 / 5-+-, 4---$ and so on and so forth. There is no way to predict the $n$ value of the present generation so that we do not know where to stop producing fractions but it seems that there is abundant fractionalization in the odd denominator regime. We try the next odd integer, $m=3$. Then (7) gives $v=1 / 3$ and (8) gives $2 / 7+$ and $2 / 5$ - for $n=1$ and for $n=2$, we get, $5 / 17++, 5 / 13+-, 3 / 7--, 3 / 11-+$ and for $n=3, p_{1}=p_{2}=p_{3}=2$ we have12/41+++, 12/31++-, 8/29+-+, 8/19+--, 8/27-++, 8/21-+-, 4/15--+ and 4/9---. In Fig. 1 we show the Haldane's hierarchy for $m=1$ parents. These numbers are not based on spin or orbit or Landau levels or two dimensionality or Hall effect. These are not even the random numbers. Hence these are not used to explain the quantum Hall effect. There is no particular reason why the continued fraction should be related to the physics of the problem.

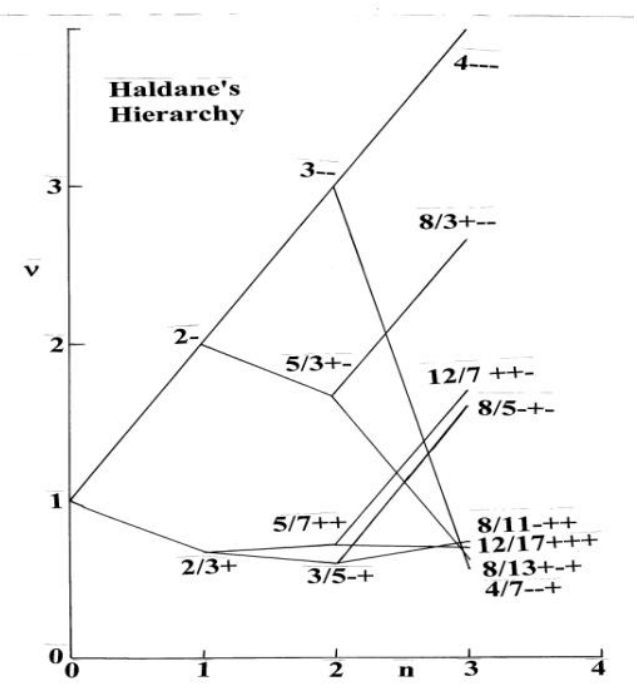

Fig. 1. Haldane's hierarchy for $v=1$.

\section{SHRIVASTAVA'S HIERARCHY}

The cyclotron frequency is defined in terms of the magnetic field as,

$$
\omega=e B / m c
$$

In the present case of single-particle current, we expect,

$$
\hbar \omega=e V_{y}
$$

This expression is completely consistent with the current (4). This is related to the flux quantization which is consistent with the charge of e, as the longitudinal resistivity does show zeroes at certain values of the magnetic field. From (9) and (10), we write,

$$
\frac{\hbar e B}{m c}=e V_{y}
$$

which upon multiplying by e/h gives,

$$
\frac{e^{2} B}{2 \pi m c}=\frac{e^{2} V_{y}}{h}
$$

which describes the current in the $\mathrm{x}$-direction so that upon taking into account the gyromagnetic ratio, we find, $I_{x}=$ $\frac{1}{2} g \frac{e^{2} B}{2 \pi m c}=\frac{1}{2} \frac{g e^{2} V_{y}}{h}$. For $\mathrm{L}=0, \mathrm{~g}=2$, it reduces to

$$
I_{x}=\frac{e^{2} V_{y}}{h}
$$

which describes the correctly quantized current for $v=1$. Hence (10) is consistent with (13). We examine the width of the plateau in the transverse resistivity in field units as a function of temperature from the experimental measurements of Hall effect in GaAs/AlGaAs heterostructures. We find that for $v=1$ the width $\delta B_{v}$ approaches zero at $T_{c 1}=6.5 \pm 0.5 \mathrm{~K}$. Similarly, for $v=2 / 3, T_{c 2 / 3}=1.1 \pm 0.1 \mathrm{~K}$ and for $v=1 / 3$ we obtain $T_{c 1 / 3}=1.7 \pm 0.2 \mathrm{~K}$. Corresponding to every fraction there is a transition. We consider the spin as well as the orbital motion, so that,

$$
g_{j} j=g_{s} s+g_{l} l=(1 / 2)\left(g_{l}+g_{s}\right) j+(1 / 2)\left(g_{l^{-}} g_{s}\right)(l-s)
$$

Multiplying both sides of this equation by $j=l+s$ and taking eigen values we find,

$$
g_{j} j(j+1)=(1 / 2)\left(g_{l}+g_{\mathrm{s}}\right) j(j+1)+(1 / 2)\left(g_{l^{-}} g \mathrm{~s}\right)[l(l+1)-s(s+1)]
$$

which upon substituting $s=1 / 2$ gives,

$$
g_{j}=g_{l} \pm \frac{g_{s}-g_{l}}{2 l+1}
$$

for $j=l \pm 1 / 2$. For $g_{\mathrm{s}}=2, g_{l}=1$ we find.

$$
g_{ \pm}=1 \pm \frac{1}{2 l+1}
$$

This is the most important relation which is the correct relation for the understanding of fractions which are observed in the quantum Hall effect experiments. In fact it is not necessary to use the relations (14)-(17) because, an alternative proof exists. The value of (17) is exactly the same as,

$$
g=\frac{2 j+1}{2 l+1}
$$


where $j=l \pm s$. Note that usually only the plus sign is conserved in the Dirac Hamiltonian. A slight modification shows that both signs are correct. That is done by introducing the negative spin which was not done by Dirac but was studied in later years. We assume the Landau level quantum number $n=1$. For $l=0, g_{+}=2, g_{-}=0$ so that we find $v=\frac{1}{2} g=1$ and 0 . For $l=1, s=1 / 2$, negative sign, $g-=2 / 3$ so that $v=1 / 3$ and for $l=1, s=1 / 2$, positive sign gives $v=2 / 3$. All of the 101 fractions found from the formula turn out to be the same as in the experimental data subject to the condition that all of the fractions for spin 1/2 are tabulated first. These are found in the Stormer's experimental data. We must postulate two-particle states to explain some of the data and some are resonances. When all of these fractions are considered, there emerges the concept of single-particle plateaus as well as "electrons clusters" for which the spin is different from $1 / 2$. Due to polarization, the cluster spin can be quite large. The hierarchy produced from (18) for both signs is given in Fig. 1 on the right hand side picture. The starting mother has $l=0$, the daughter has $l=1$, the grand daughter has $l=2$, etc which builds the family and the clusters of families [7].

\section{THE DIRAC EQUATION}

The Dirac equation, obtained from the Schroedinger equation by using the space-time symmetry and by linearizing the momentum is given by,

$$
\left(c \alpha \cdot p+\beta m c^{2}\right) \Psi(x, t)=i \hbar \frac{\partial}{\partial t} \Psi(x, t)
$$

where $m$ is the rest mass of the electron, $p$ is the linear momentum, $\mathrm{c}$ is the velocity of light and $\alpha_{x}, \alpha_{y}, \alpha_{z}$ and $\beta$ are anticommuting $4 \times 4$ Dirac matrices. The free-particle energy solutions are,

$$
E_{ \pm}(p)= \pm\left(c^{2} p^{2}+m^{2} c^{4}\right)^{1 / 2}
$$

where positive sign is associated with the electron and negative energy solutions correspond to the positron. A classical electron moving in a circular orbit has an orbital angular momentum, $L=r \times p$ and a magnetic dipole moment, $\mu=-e r \times v / 2$, where $r$ is the coordinate of the electron, $\mathrm{p}$ its linear momentum, $\mathrm{v}$ is the velocity and $\mathrm{e}$ is the charge. Therefore, the ratio of the magnetic moment to the dipole moment is $\gamma=\mu / L=-e / 2 m$. Since the electron has spin, it produces a correction to the ratio of the magnetic moment to the dipole moment so that the corrected value becomes $\gamma=-g e / 2 m$. Actually, the Dirac equation does not conserve angular momentum unless spin is introduced. Therefore, spin effects are assigned to the Dirac equation. The spin angular momentum is $\hbar / 2$. The electron is a charged particle so that electromagnetic field is associated with it. The correction to the $g$ value due to this effect is given in terms of the fine structure constant as $g=2(1+\quad \alpha / 2 \pi)$, where $\alpha=e^{2} / \hbar c=1 / 137.03599976(50)$. The electromagnetic correction to the $g$ value is written as $g-2=\alpha / \pi=0.0023193043737(82)$. In this way, the $g$ value is related to the charge of the electron. Later on, we will discover that $g$ values describe the fractional charge. It is possible to argue that $\hbar$ and $\mathrm{c}$ are constants so that only the charge of the particle is affected by the angular momentum. The unit of resistivity may be fixed by $h / e^{2}=\mu_{o} c / 2 \alpha=25812.807572(95)$ ohms, with $\mu_{0}=4 \pi \times 10^{-7} N A^{-2}$ exactly with $c$ in units of $\mathrm{m} / \mathrm{s}$. This means that the Dirac equation predicts spin which enters in the ratio of magnetic moment to angular momentum in the form of a $g$ value which can be described in terms of $\mathrm{e}, \mathrm{h}$ and $\mathrm{c}$ to great accuracy. The speed of light is now known exactly, $c=2.99792458 \times 10^{10}$ $\mathrm{cm} / \mathrm{s}$. We write, $g=\frac{2 j+1}{2 l+1}$ which for $j=l \pm s$ gives $\frac{1}{2} g=\frac{l+(1 / 2) \pm s}{2 l+1}$. In the expression for energy in a magnetic field we have H.S so that $S_{z}$ can give a factor of $1 / 2$.

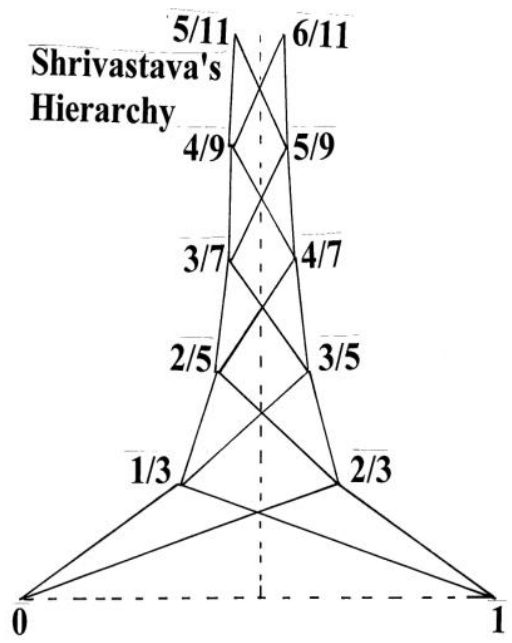

Fig. 2. Shrivastava's hierarchy which explains the experimental data correctly.

We consider the spin as well as the orbital motion so that,

$$
g_{j} j=g_{s} s+g_{l} l=\frac{1}{2}\left(g_{l}+g_{\mathrm{s}}\right) \mathrm{j}+\frac{1}{2}\left(g_{l^{-}} g_{\mathrm{s}}\right)(l-s)
$$

We include the bound electrons as well as the free electrons which have finite orbital angular momentum quantum numbers. Multiplying both sides of the above equation by $j=l+s$ and taking eigen values, we find,

$$
g_{j} j(j+1)=\frac{1}{2}\left(g_{l}+g_{s}\right) j(j+1)+\frac{1}{2}\left(g_{1} g_{s}\right)[l(l+1)-s(s+1)] .
$$

Multiplying $j$ by $j$ gives $j^{2}$ which we write as $j(j+1)$. Similarly, $(l-s)(l+s)=l^{2}-\mathrm{s}^{2}=l(l+1)-s(s+1)$. In this equality at $l=s$, the first bracket, the second value as well as the $l(l+1)-s(s+1)$ are all zero so that we obtain $0=0$ but such a situation never arises since $\mathrm{s}$ is half integer and $l$ is integer. Upon substituting $s=1 / 2$, we get,

$$
g_{j}=g_{l} \pm \frac{g_{s}-g_{l}}{2 l+1}
$$


for $j=l \pm \frac{1}{2}$. For $g_{\mathrm{s}}=2, g_{l}=1$, we find,

$$
g_{ \pm}=1 \pm \frac{1}{2 l+1}
$$

or,

$$
g_{ \pm}=\frac{2 l+1 \pm 1}{2 l+1}
$$

For $s=-1 / 2$, (note the - sign) the $g$ value is,

$$
g=2 l l(2 l+1)
$$

and for $s=+1 / 2$, (note the + sign) it is,

$$
g_{+}=\frac{2(l+1)}{2 l+1}
$$

We introduce the negative as well as the positive spin so that,

$$
\frac{1}{2} g_{ \pm}=\frac{l+(1 / 2) \pm s}{2 l+1}
$$

The Zeeman Hamiltonian is written as $H=\mathrm{g} \mu_{B} B . J$ so that the resonance frequency is $\hbar \omega_{c}=g \mu_{B} B$ where the Bohr magneton is $\mu_{B}=e \hbar / 2 m c$ so that the cyclotron frequency is,

$$
\omega_{c}=(1 / 2) g_{ \pm} e B / m c
$$

Hence the charge e can be replaced by an effective charge,

$$
e_{e f f}=\frac{1}{2} g_{ \pm} e
$$

For negative spin, $s=-1 / 2$, and $l=0$, the expression (28) gives, $(1 / 2) g_{+}=0$, which shows that there are particles of zero charge, and for positive spin, $s=+1 / 2, l=0, e_{e f f}=e$, predicts a charge of $e_{e f f}=1$ in units of electron charge. This means that there are electrons with the usual charge, e. For $s=-1 / 2, l=1$, the charge is $1 / 3$ and for $s=+1 / 2, l=1$, the charge is $2 / 3$. Thus the electron splits into fractionally charged particles. In fact, all of these values are the same as those measured in the experimental work of Stormer. In actual semiconductor heterostructures, there are electron clusters so that higher values of the spin such as $3 / 2$ and $5 / 2$ also become allowed and the spin projection quantum number $\mathrm{s}_{\mathrm{z}}$ may be equal to $1 / 2$ or higher so that, instead of $(1 / 2) g_{ \pm}$, other values such as $(3 / 2) g_{ \pm}$will arise. This opens the possibility of a large number of spin states.

\section{THE DiRAC MATRICES}

The Dirac equation describes particles of charges -e and $+e$ which are called the electron and the positron, respectively. When $\beta \mathrm{mc}^{2}$ term is left out, fermions of zero mass are produced. In our calculation, since there are two $g$ values, $g_{ \pm}$, there are four eigen values for spin $1 / 2$ instead of 2 . Two values occur for spin $1 / 2$ with $g_{+}$and two more values occur for spin $1 / 2$ with $g$. All of these four values belong to the electron. If positrons are also considered, there will be 8 eigen values. Considering the electron states only, the matrix we introduce is,

$$
M=\left(\begin{array}{cccc}
(1 / 2) g_{+} \mu_{B} H & 0 & 0 & 0 \\
0 & (1 / 2) g_{-} \mu_{B} H & 0 & 0 \\
0 & 0 & -(1 / 2) g_{+} \mu_{B} H & 0 \\
0 & 0 & 0 & -(1 / 2) g_{-} \mu_{B} H
\end{array}\right)
$$

so that the Dirac equation with electron states only appears as,

$$
\left(\beta m c^{2}+c \alpha \cdot p\right) \psi=E \psi+M \psi
$$

where,

$$
\begin{gathered}
\alpha_{1}=\left(\begin{array}{cccc}
0 & 0 & 0 & 1 \\
0 & 0 & 1 & 0 \\
0 & 1 & 0 & 0 \\
1 & 0 & 0 & 0
\end{array}\right), \alpha_{2}=\left(\begin{array}{cccc}
0 & 0 & 0 & -i \\
0 & 0 & i & 0 \\
0 & -i & 0 & 0 \\
i & 0 & 0 & 0
\end{array}\right), \\
\alpha_{3}=\left(\begin{array}{cccc}
0 & 0 & 1 & 0 \\
0 & 0 & 0 & -1 \\
1 & 0 & 0 & 0 \\
0 & -1 & 0 & 0
\end{array}\right), \beta=\gamma_{4}=\left(\begin{array}{cccc}
1 & 0 & 0 & 0 \\
0 & 1 & 0 & 0 \\
0 & 0 & -1 & 0 \\
0 & 0 & 0 & -1
\end{array}\right) .
\end{gathered}
$$

Thus we find that there is a new anticommutator,

$$
\alpha_{1} M+M \alpha_{1}=G_{1}
$$

where,

$$
G_{1}=\left(\begin{array}{cccc}
0 & 0 & 0 & (1 / 2) g_{o} \\
0 & 0 & -(1 / 2) g_{o} & 0 \\
0 & -(1 / 2) g_{o} & 0 & 0 \\
(1 / 2) g_{o} & 0 & 0 & 0
\end{array}\right)
$$

with $g_{0}=g_{+}-g_{-}=\frac{2 s}{2 l+1}$. For $l=0,(1 / 2) g_{0}=s$ so that $G_{1}$ becomes a spin only matrix,

$$
G_{1}(l=0)=\left(\begin{array}{cccc}
0 & 0 & 0 & s \\
0 & 0 & -s & 0 \\
0 & -s & 0 & 0 \\
s & 0 & 0 & 0
\end{array}\right)
$$

Similarly,

$$
\alpha_{2} M+M \alpha_{2}=G_{2}
$$

$$
G_{2}=\left(\begin{array}{cccc}
0 & 0 & 0 & -(i / 2) g_{o} \\
0 & 0 & -(i / 2) g_{o} & 0 \\
0 & (i / 2) g_{o} & 0 & 0 \\
(i / 2) g_{o} & 0 & 0 & 0
\end{array}\right)
$$


and at $l=0$ we obtain the spin only matrix,

$$
G_{2}(l=0)=\left(\begin{array}{cccc}
0 & 0 & 0 & -i s \\
0 & 0 & -i s & 0 \\
0 & i s & 0 & 0 \\
i s & 0 & 0 & 0
\end{array}\right)
$$

and the anticommutator with $\alpha_{3}$ is independent of $l$,

$$
\alpha_{3} M+M \alpha_{3}=0
$$

Thus the matrix $M$ which gives the $4 \times 4$ matrix for $s=1 / 2$ and anticommutes with the Dirac matrices except,

$$
\beta M-M \beta=0
$$

which is a commutator. In this way we find the commutation properties of the new matrices. When the dimensions are $4 \times$ 4 for the electron only, another $4 \times 4$ elements are needed for the positron so that if it gives the electrons as well as the positrons, it will have to be a $8 \times 8$ matrix. Whereas, Pauli used only $2 \times 2$ matrices for the electron, they became $4 \times 4$ Dirac matrices due to space time symmetry, we have to use $8 \times 8$ matrices because of $\mathrm{s}$ as well as $-\mathrm{s}$ for the electron as well as for the positron.

For the charged particles in an electromagnetic field the Dirac hamiltonian is changed to,

$$
H=\beta m c^{2}+c \alpha \cdot\left(p-\frac{e}{c} A\right)+e \varphi(x, t)
$$

where $A$ is the vector potential of the electromagnetic field, which reduces the linear momentum and $\varphi$ is the scalar potential. The wave function is four dimensional but it is sufficient to take only two at a time,

$$
\left[\frac{1}{2 m} \sum_{j}\left|p-\frac{e}{c} A(x, t)\right|^{2}-\frac{e \hbar}{2 m c} \sum_{j} \sigma_{j} B_{j}(x)\right] \psi=\left(E-m c^{2}\right) \psi .
$$

Therefore, the magnetic moment of the particle is et/2mc. The magnetic moment of the proton calculated by using the proton mass does not agree with the experimental value. Therefore, it is necessary to correct the expression for the magnetic moment. Accordingly,

$$
\mu_{\text {proton }}=\frac{g_{p} \hbar e}{2 m c}
$$

with $g_{p}=5.58$ for the proton. Similarly, there is a need to introduce the $\mathrm{g}$ value for the neutron, $g_{n}=-3.826$ which gives the correct value of neutron magnetic moment. According to (23), the magnetic moment consists of a spin part and an orbital part. The spin and orbital gyromagnetic factors are $g_{l}=1, g_{s}=5.585$ for the proton and $g_{l}=0, g_{s}=-3.826$ for the neutron. The magnetic moment of the proton for negative sign, $j=l-(1 / 2)$ is $\mu_{p}=[1-2.29 /(j+1)] j$ and for the positive sign, $j=l+(1 / 2), \mu_{p}=j+2.29$. Similarly, for the neutron, $j=l-(1 / 2)$, $\mu_{n}=[1.91 /(j+1)] j$ and for $j=l+(1 / 2), \mu_{n}=-1.91$. The expression (23), thus gives the correct values for the magnetic moments of the proton and the neutron. When Lande first introduced the splitting factor, it had only one value,

$$
g=1+\frac{J(J+1)-L(L+1)+S(S+1)}{2 J(J+1)}
$$

but according to (23) there are two separate values, one for the spin and the other for the orbit. In the case of the electron and the positron very accurate values of $(g-2) / 2$ are available but only for the $l=0$ state so that only one value is measured. Many different orbital states are not available by this method. According to us a whole series of values arise as we change the value of the orbital angular momentum. The free electron with $g=2$ has only one value for $g$ and hence only one value for the magnetic moment but we find a series of values. Changing the value of $l$ leads to a change in $s$ such that the angular momentum is conserved but this type of phenomenon has not been discussed in the literature. In particular, fractions such as those given by (26) and (27) lead to a fractional charge given by (30). If we ignore the correction to the charge, we must correct the mass so that the correct cyclotron frequency is obtained,

$$
\omega_{c}=\frac{e B}{c\left[m /(1 / 2) g_{ \pm}\right]}
$$

Not only that the mass of the electron is enhanced but there are double the number of values. For $(1 / 2) g=1 / 3$, the mass must be replaced by $3 \mathrm{~m}$ and for $(1 / 2) g_{+}=2 / 3$, the mass becomes $3 \mathrm{~m} / 2$. Therefore, either the charge or the mass completely changes compared with the free electron value.

\section{DIRAC POINTS}

The crossing points in the band structure are often called Dirac points particularly when the energy versus wave vector looks almost a straight line. In the Hamiltonian there is the usual kinetic energy which is $\mathrm{k}^{2} / 2 \mathrm{~m}$ so that the apparently looking straight lines are parabolic. In any case, the crossing points have been named Dirac points. In our calculation these points often have a small gap and these are Schroedinger points. In our Dirac equation there is no similarity with Novoselov's Dirac points [9], [10] which are interesting by themselves. A linearization of the bands may be a nice simplification.

\section{CONCLUSION}

The hierarchy of states in the quantum Hall effect is built by changing the angular momentum. In the Haldane's work, the hierarchy is made by a random number not matched with the data. There is effect on the Dirac equation in terms of increased eigen values because of the two signs of spin in the total angular momentum. The entire data can be explained by two signs in the $g$ values. The key formula that emerges has double the number of eigen values. All our calculated values agree with Stormer's data [8]. 


\section{REFERENCES}

[1] K. N. Shrivastava, "The quantum Hall effecr: Interpretation of the experimental data," International Journal of Applied Physics and Mathematics, vol. 2, no. 6, pp. 467-473, November 2012.

[2] K. N. Shrivastava, "The quantum Hall effecr: Helicity, graphite and grapheme," International Journal of Applied Physics and Mathematics, vol. 3, no. 1, pp. 1467-22473, January 2013.

[3] K. N. Shrivastava, "Universal introduction to the quantum Hall effecr," International Journal of Applied Physics and Mathematics, vol. 3, no. 3, pp. 208-216, May 2013.

[4] K. von Klitzing, G. Dorda, and M. Pepper, "New method for high-accuracy determination of the fine structure constant," Phys. Rev. Lett., vol. 45, pp. 494-497, August 11, 1980.

[5] D. C. Tsui, H. L. Stormer, and A. C. Gossard, "Two-dimensional magnetotransport in the extreme quantum limit," Phys. Rev. Lett., vol. 48, pp. $1559-1562,1982$.

[6] F. D. M. Haldane, "Fractional quantization of the Hall effect: A hierarchy of incompressible quantum fluid states," Phys. Rev. Lett., vol. 51, pp. 605-608, August 1983.

[7] K. N. Shrivastava, "The quantum Hall effect:The symmetry and the velocity, (IOP)," J.Phys:Conf. Ser., vol. 423, article number: 012003, 2013.

[8] H. L. Stormer, "Nobel lecture: The fractional quantum Hall effect," Reviews of the Modern Physics, vol. 71, pp. 875-889, July 1999.

[9] A. Luican, Q. Li, A. Raina, J. Kong, R.R. Nair, K. S. Novoselov, A. K. Geim, and E. Y. Andrei, "Single layer behavior and its breakdown in twisted graphene layers," Physical. Review. Letters, vol. 106, no. 12, article number: 126802, March 2012.

[10] D. A. Abanin, K. S. Novoselov, U. Zeiller, P. A. Lee, A. K. Geim, and L. S. Levetov, "Dissipative quantum Hall effect in graphene near the Dirac point," Physical Review Letters, vol. 98, article number: 196806 , May 2007.

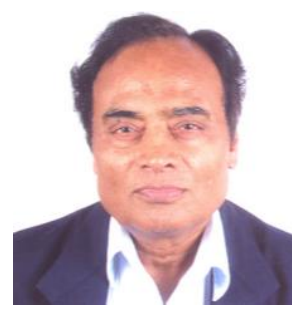

Keshav N. Shrivastava was born on July 11, 1943. He obtained his B.Sc. degree from the Agra University in 1961, M.Sc. degree from the University of Allahabad in 1963, Ph.D. from the Indian Institute of Technology Kanpur in 1966 and D.Sc. from Calcutta University in 1980.

$\mathrm{He}$ worked at the Clark Universityin USA during 1966-68, at the Harvard University USA during the summer of 1968, at the Montana State University USA during 1968-69, and at the University of Nottingham during 1969-70. He was Associate Professor at the Himachal Pradesh University Simla, India during 1970-74. He worked at the University of California Santa Barbara USA during 1974-75 and at the State University Utrecht, the Netherlands, during 1975-76. He was Professor at the University of Hyderabad where he worked from 1978 till 2005. He worked in Tohoku University Japan during 1999-2000. He visited the University of Zurich Switzerland for short times during 1984, 1985, 1988 and 1999. He worked in the University of Houston USA in the summer of 1990. He visited the Royal Institute of Technology Stockholm Sweden and the University of Uppsala Sweden during 1994. He visited the Technical University of Vienna, Austria in the summer of 1995 . He visited the Princeton University USA and the University of Cincinnati USA during 2003. He was Professor in the University of Malaya Kuala Lumpur Malaysia during 2005-2011. He is affiliated with the University of Hyderabad, India. He has worked in electron spin resonance, spin-phonon interaction, relaxation, Moessbauer effect, exchange interaction, superconductivity and the quantum Hall effect. He discovered the flux quantized energy levels in superconductors, the spin dependent flux and the correct theory of the quantum Hall effect. He has published more than 232 papers in journals and he is the author of the following books. 1) Superconductivity: Elementary Topics, World Scientific Singapore 2000. 2) Introduction to Quantum Hall effect, Nova Sci. Pub N.Y. 2002 and 3) The quantum Hall effect: Expressions, Nova Sci. N.Y. 2005.

Prof. Shrivastava is a Fellow of the Institute of Physics London, Fellow of the National Academy of Sciences India and member of the American Physical Society. 